\title{
What is fluidity? Designing an experimental system to probe stress and velocity fluctuations in flowing suspensions
}

\author{
Marcel Workamp ${ }^{1}$, Sepideh Alaie ${ }^{1}$, and Joshua A. Dijksman ${ }^{1 \star}$ \\ ${ }^{1}$ Physical Chemistry and Soft Matter, Wageningen University \& Research, The Netherlands
}

\begin{abstract}
We develop a method to investigate the microscopic origin of granular fluidity. We design a Couette cell in which we can probe the flow of soft hydrogel suspensions. As we drive the suspension with a rheometer, we have access to global flow characteristics. In addition, the Couette cell has been modified to have a transparent bottom and lid, allowing for imaging of suspension characteristics in transmission, for example flow fields. We can also use transmission imaging to probe local stresses in the suspension: we use hydrogel particles composed of gelatin, which through its photoelastic properties gives access to local stress fluctuations. We thus have access to all local microscopic variables that are relevant in the understanding of granular suspensions. We show here that our setup can indeed visualize stress fields inside the suspensions and perform flow field measurements in transmission mode. We compare the profiles of the gelatin suspension to that of a polyacrylamide hydrogel suspension, to assess robustness of observed phenomenology. We find that the flow profiles for both types of hydrogels are different; gelatin suspensions feature narrower and less rate-dependent flow profiles. We speculate on the origin of the observed difference by considering the frictional properties of the suspended particles.
\end{abstract}

\section{Introduction}

The flow behavior of dry granular materials and dense suspensions can generally be viewed as consisting of two regimes. In the slow flow limit or quasi-static regime, grain-to-grain interactions are frictional and the shear stress is rate-independent. At higher driving rates, the material becomes more fluid-like: inertia or the viscosity of the interstitial fluid starts to play a role and the stress increases are well described by a power law originating from collisional or viscous energy loss in this flow regime. Such behaviour can be captured by the Herschel-Bulkley (HB) model that describes yield-stress fluids:

$$
\tau=\tau_{0}+k \dot{\gamma}^{n}
$$

where $\tau$ denotes the shear stress, $\tau_{0}$ the yield stress, $\dot{\gamma}$ the shear rate, $k$ a proportionality constant and $n$ a power law index. The HB model is very effective in capturing the macroscopic flow response of granular materials and suspensions. However, to understand and describe the local flow behavior of these materials it has been shown to be ineffective. In particular, it is well-known that HB fluids can form shear bands (for a review see Ref. [1]), especially when a spatially inhomogeneous stress field is applied. One way to apply such a stress field is in a Couette geometry, where a cylinder rotates coaxially in a second cylinder. The shear stress $\tau$ in such a system decays as $\sim 1 / R^{2}$, with $R$ the radius. Although one may expect the

\footnotetext{
^e-mail: joshua.dijksman @ wur.nl
}

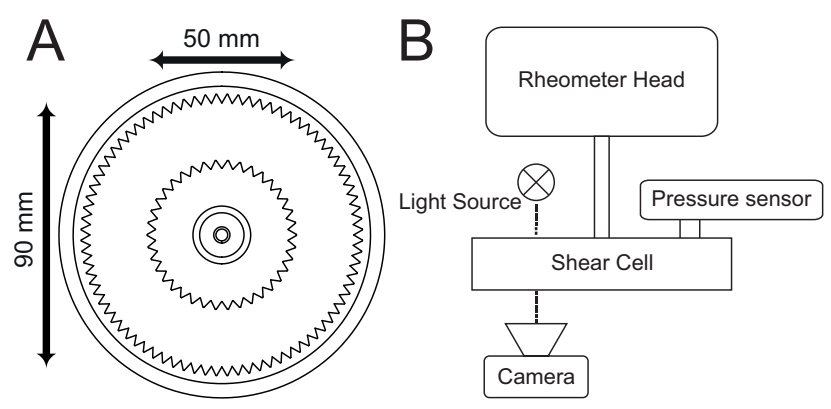

Figure 1. (A) Schematic of the Couette cell. The cell has a height of $20 \mathrm{~mm}$. (B) Schematic overview of the experimental setup. A pressure sensor is mounted to the lid of the cell. We image the suspension in transmission mode using an LED lamp and diffuser, and an 8-bit camera.

flow to stop abruptly at $R\left(\tau<\tau_{0}\right)$, the flow profile for a yield stress fluid is smooth [1-4].

We can view flows of granular materials in terms of two dimensionless quantities. A ratio of two time scales provides us with $I=\dot{\gamma} d \sqrt{\rho_{p} / P}$, with $d$ the particles' diameter, $\rho_{p}$ its density and $P$ the local pressure. The rheology is then described by the friction coefficient $\mu=\tau / P$ to be a simple function of $I$ [5]. This theory can be extended to explain the rheology of suspensions [6]. However, such local descriptions fail to accurately describe flows in the quasistatic regime, such as the smoothly decaying velocity 

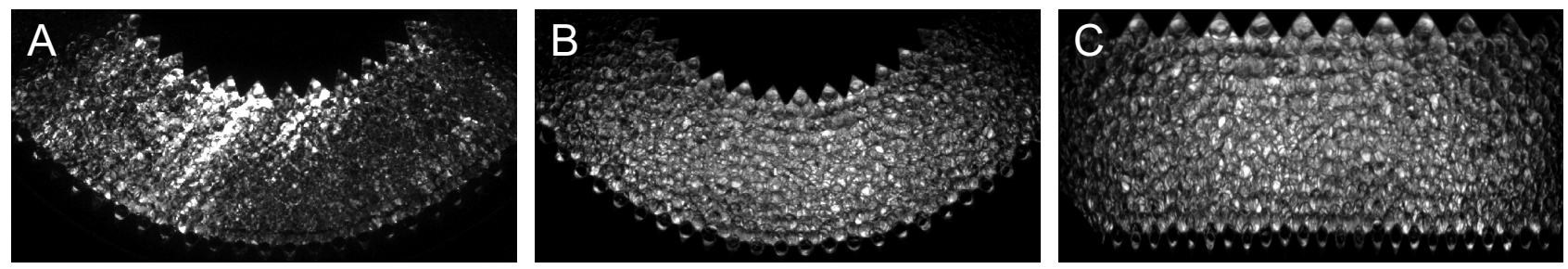

Figure 2. (A) Example of an image of the photoelastic response of the gelatin particles. Stresses are anisotropic and point against the direction of shear (inner cylinder rotating clockwise). (B) Image of a suspension without polarizers. (C) Same as (B) after transformation of the coordinate system.

field discussed before. To solve these issues, a fluidity theory first proposed for emulsions [7], was adapted to granular materials [8]. An additional so-called granular fluidity field $g$ may be used to predict nonlocal effects in multiple geometries $[8,9]$. Fluidity modeling requires remarkably few fitting parameters, yet the microscopic meaning of the fluidity field is as yet subject of debate. The fluidity field somehow models the fluctuations present in even a slowly flowing granular material, yet are these fluctuations of velocities, stresses, or something else?

Here we propose an experimental environment in which we can probe the role of local stress and velocity fluctuations in the flow behavior of a three-dimensional suspension. We perform experiments in Couette flows of gelatin particles. The gap of the Couette cell is approximately 10 particle diameters wide (see Section 2), resulting in a highly heterogeneous stress field, making nonlocal effects probable. Gelatin is photoelastic [10], giving us access to local stress fluctuations, by imaging the particles in transmission mode. As the particles are transparent but not refractive index matched, we also have access to the velocity profiles of the particles. In this paper we will demonstrate that we can indeed optically resolve an average local stress field inside a flowing suspension. Since particle image velocimetry (PIV) of granular materials is typically done by imaging only a surface, it is not evident that transmission mode imaging can also resolve flow fields. In the second part of this paper we show that we can indeed obtain velocity measurements from transmission imaging. To see how universal the flow profiles of the gelatin suspension are, we then compare them to those of another typical hydrogel, polyacrylamide.

\section{Materials \& Methods}

\subsection{Experimental setup}

We use a custom Couette geometry with fixed volume to drive the flow of a hydrogel particle suspension. The inner cylinder diameter $d_{i}=50 \mathrm{~mm}$, while the outer cylinder diameter $d_{o}=90 \mathrm{~mm}$ (Figure 1A). To avoid slip, both inner and outer walls are made rough with teeth of approximately $2.5 \mathrm{~mm}$. The height of the shear cell $h=20 \mathrm{~mm}$. The bottom and top plate of the cell are made of smooth acrylic glass. We measure the vertical confining pressure using a load cell on part of the top plate. We use a stresscontrolled rheometer (Anton-Paar MCR 300) to drive the inner cylinder at a fixed rotation rate. We image the suspension from below with an 8-bit camera, while illuminating from the top using an array of LEDs and a diffuser (Figure 1B). We can add polarizers to image the photoelastic response, of which we show an example in Figure 2A.

\subsection{Hydrogel particles}

We synthesize spherical hydrogel beads of roughly $2 \mathrm{~mm}$ in diameter to perform the experiments. We use a method described in detail elsewhere [11]. We use two different materials; gelatin and polyacrylamide (PAAm). In short, the gelatin particles are made by dispersing a hot solution of gelatin $(10 \mathrm{wt} \%)$ in a column of cold oil (decane), thereby solidifying the droplets. The resulting particles are cross-linked using glutaraldehyde [12] to avoid dissolution of the gelatin when they are suspended in water for the flow experiments. The PAAm particles are made in a similar way: we disperse a solution of acrylamide (20 wt \%), bis-acrylamide (1 wt \%) and ammonium persulfate $(1 \mathrm{wt} \%)$ in a column of oil (kerosene) with a catalyst (TEMED, 2 wt \%). As we have control over the droplet size, we can make the suspension bidisperse to avoid crystallization. We have measured the Young's modulus of

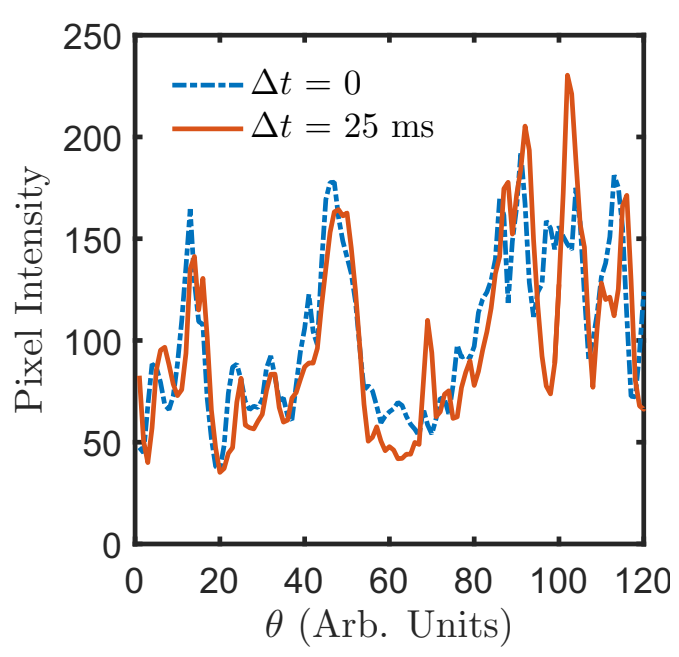

Figure 3. Intensity profiles of a row of pixels at two distinct times, showing that the signal is correlated. The profile at later time is shifted to the right. 

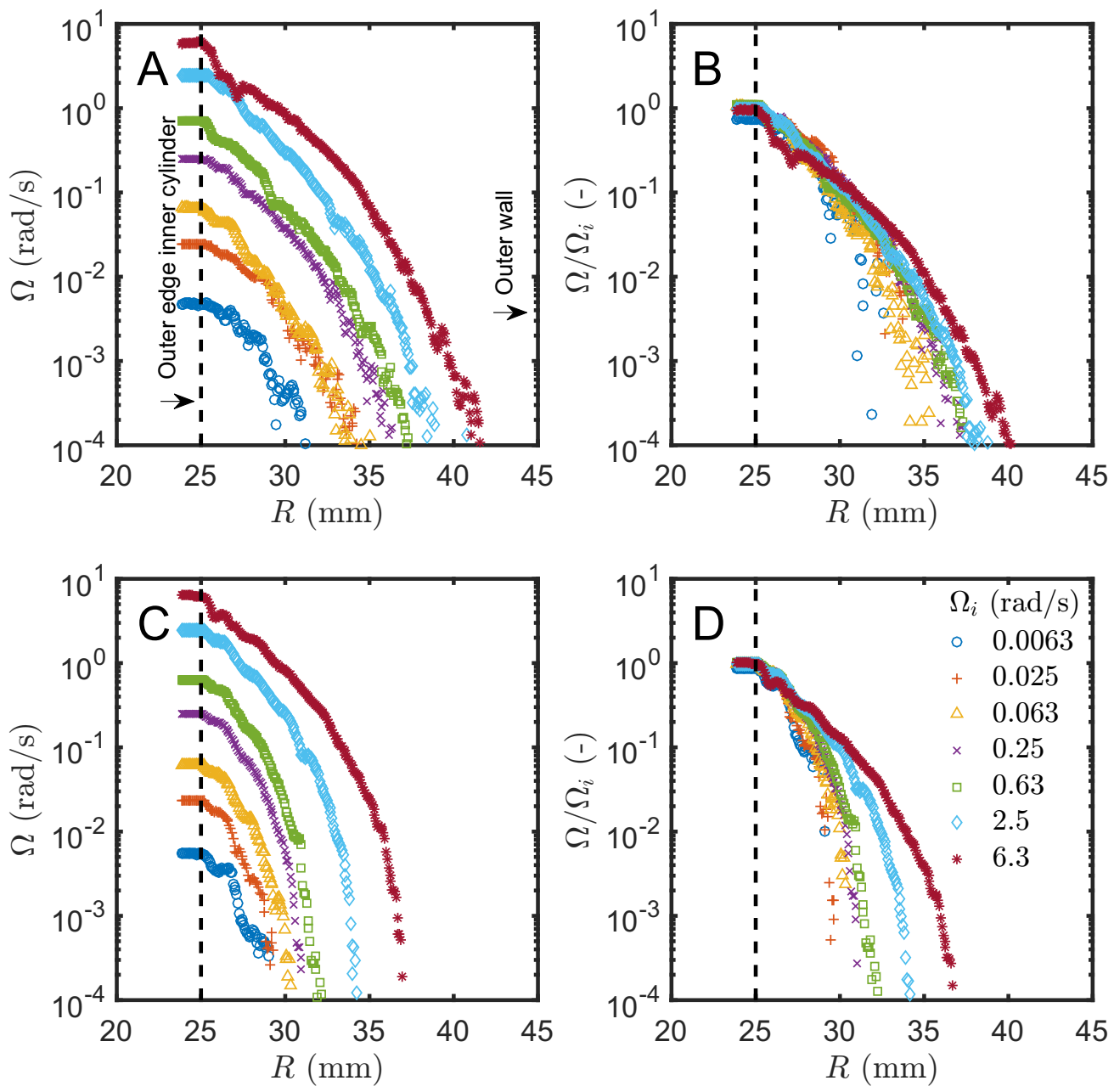

Figure 4. (A,B) Flow profiles for the PAAm particles. (C,D) Flow profiles for the gelatin particles. Legend applies to all panels.

both particle types to be approx. $3 \times 10^{5} \mathrm{~Pa}$. The particles thus differ only in their chemical composition, which may result in differences in their surface structure or other physico-chemical properties.

\subsection{Experimental procedure}

We perform experiments in suspensions at fixed volume fraction, characterized by the pressure exerted on the top plate. We measure this confining pressure at a low rotation rate of the inner cylinder $\left(\Omega_{i}=6.3 \times 10^{-3} \mathrm{rad} / \mathrm{s}\right)$ and make sure all experiments are carried out at a pressure $P^{p} \approx 200 \mathrm{~Pa}$. As the particles are porous and their swelling depends on the solvent, density matching of these particles is challenging. We thus use water as the interstitial fluid. The maximum hydrostatic pressure resulting from the density difference $\Delta \rho$ between the particles and water can be estimated $P_{g}=\Delta \rho g h \approx 20 \mathrm{~Pa}$. The pressure due to the density mismatch is always much smaller than any applied confining pressure, so we can neglect the role of $P_{g}$. Before our measurements, we pre-shear the suspension at $\Omega_{i}=62 \mathrm{rad} / \mathrm{s}$ for 10 seconds. After waiting for 30 seconds, we then start driving the suspension at a fixed $\Omega_{i}$ ranging from $6.3 \times 10^{-3}-6.3 \mathrm{rad} / \mathrm{s}$, thus spanning three decades in driving rate. For each rate, we image the suspension for 10 seconds at 119 frames/second. To ensure that we measure in the steady-state, we start recording only after the inner cylinder has made about one full rotation.

\subsection{Particle Image Velocimetry}

We first convert our recorded images such that the coordinates are transformed from Cartesian to polar (Figure $2 \mathrm{~B}+\mathrm{C}$ ). This allows for finding the intensity as a function of the angle $\theta$ at given radius $R$, corresponding to a row of pixels. Typical for PIV, we find the correlation between intensity profiles to get an estimate of the velocity at given $R$. Although we image in transmission mode, we can still find significant correlation between intensity profiles; an example is shown in Figure 3. By finding the shift of the signal for different time steps $\Delta t$, we find the angular velocity $\Omega(R)$ - this is the standard method to do PIV.

\section{Results \& Discussion}

We plot the results of our analysis in Figure 4. In Figure $4 \mathrm{~A}$, we plot the velocity profiles of the PAAm suspen- 
sion, showing that the shear band widens as we increase the driving rate of the inner cylinder $\Omega_{i}$, as expected and similar to the findings in Ref. [13, 14]. For the gelatin suspension (Figure 4C), the shear is much more localized; particles in the outer half of the gap show very little movement, even at the highest rates applied. This finding shows that hydrogel material properties affect flow properties in this dense limit. We can find another difference in Figure $4 \mathrm{~B}+\mathrm{D}$, where we have normalized the angular velocity $\Omega$ with the applied driving rate $\Omega_{i}$. For the PAAm suspension (Figure 4B), the width of the shear band increases as $\Omega_{i}$ increases. However for the gelatin suspension (Figure 4D), the shear band has very similar width, except for the two highest rates. We believe the differences in flow profiles between these two similar materials to be related to their frictional behavior. It is known that hydrogels may have very complex frictional behavior [15], where the friction coefficient may depend on the load as well as the sliding velocity. Gel friction depends on the chemical structure of the gel [16]. Thus it is likely that the two types of gel examined here have different frictional behavior, resulting in different flow profiles.

\section{Conclusion}

We demonstrate that we can successfully measure stress fields and perform PIV on suspensions of hydrogel particles in transmission mode, using a custom Couette cell. We find that, although composed of very similar particles, gelatin and polyacrylamide suspensions have different flow profiles. We speculate on the origin of the observed difference by considering the frictional properties of the suspended particles. The gelatin suspension is a potent new experimental system for suspension rheology, as we have access to both local stress and velocity fluctuations. As we also drive the suspension flows with a rheometer, we can measure both global and local flow characteristics, which provides an excellent test bed for the fluidity model.

\section{References}

[1] P. Schall, M. van Hecke, Annual Review of Fluid Mechanics 42, 67 (2009)

[2] P. Coussot, J. Raynaud, F. Bertrand, P. Moucheront, J. Guilbaud, H. Huynh, S. Jarny, D. Lesueur, Physical review letters 88, 218301 (2002)

[3] G. Ovarlez, S. Rodts, A. Ragouilliaux, P. Coussot, J. Goyon, A. Colin, Physical Review E 78, 036307 (2008)

[4] H. De Cagny, A. Fall, M.M. Denn, D. Bonn, Journal of Rheology (1978-present) 59, 957 (2015)

[5] F. da Cruz, S. Emam, M. Prochnow, J.N. Roux, F. Chevoir, Physical Review E 72, 021309 (2005)

[6] F. Boyer, É. Guazzelli, O. Pouliquen, Physical Review Letters 107, 188301 (2011)

[7] J. Goyon, A. Colin, G. Ovarlez, A. Ajdari, L. Bocquet, Nature 454, 84 (2008)

[8] K. Kamrin, G. Koval, Physical Review Letters 108, 178301 (2012)

[9] D.L. Henann, K. Kamrin, Proceedings of the National Academy of Sciences 110, 6730 (2013)

[10] A. Kuske, G.S. Robertson, Photoelastic stress analysis (Wiley-Interscience, 1974)

[11] M. Workamp, S. Alaie, J.A. Dijksman, Review of Scientific Instruments 87, 125113 (2016)

[12] A. Bigi, G. Cojazzi, S. Panzavolta, K. Rubini, N. Roveri, Biomaterials 22, 763 (2001)

[13] N. Huang, D. Bonn, Journal of fluid mechanics 590, 497 (2007)

[14] G. Koval, J.N. Roux, A. Corfdir, F. Chevoir, Physical Review E 79, 021306 (2009)

[15] J.P. Gong, Soft matter 2, 544 (2006)

[16] J.P. Gong, Y. Osada, in High Solid Dispersions (Springer, 2010), pp. 203-246 